\title{
A Challenging Cesarean Delivery and Perioperative Course in a Former Polysubstance Abuser Without the Use of Traditional Opioids
}

\author{
David A. Gutman ${ }^{\mathrm{a}, \mathrm{b}}$, Jennifer R. Matos ${ }^{\mathrm{a}}$, Christopher A. Skorke
}

\begin{abstract}
Substance abuse is a major challenge in the United States. According to the Human Resources and Services Administration, we are in an opioid crisis with over 130 people a day dying from opioid-related drug overdoses. As awareness of this epidemic has grown, there has been an increase in patients coming in for surgery requesting a narcotic-free anesthetic. This presents both a challenge and an opportunity for the anesthesiologist who has a duty to respect the patient's autonomy while simultaneously achieving the appropriate perioperative outcome. The considerations are especially important in the vulnerable population of pregnant women.
\end{abstract}

Keywords: Polysubstance abuse; Patient autonomy; Regional anesthesia; Cesarean delivery; Relapse; Recovery; Opioid sparing; Buprenorphone; Medical ethics; Quadratus lumborum

\section{Introduction}

Relapse in the recovering addict is a very real concern for the individual, the family and all those involved [1]. The first thing our patient said to us upon initial consultation was, "I'm terrified that I will re-start using and die; please help me". The patient emphatically expressed this was her greatest concern considering that "relapse is the recovered addict's biggest fear [2]". This presents a major challenge for the anesthesiologist since opioids have been the mainstay of pain management therapy for as long as they have existed. While the risk of relapse is ever present, the dangers of poor post-operative pain management is also very real and can worsen the exact situation the patient is trying to avoid through self-medication or

Manuscript submitted March 3, 2020, accepted March 12, 2020

aDepartment of Anesthesia and Perioperative Medicine, Medical University of South Carolina, Charleston, SC, USA

${ }^{\mathrm{b}}$ Corresponding Author: David A. Gutman, Department of Anesthesia and Perioperative Medicine, Shawn Jenkins Children's Hospital, 10 McClennan Banks Drive, Suite SJ2190, Charleston, SC 29425-8905, USA.

Email: Gutman@MUSC.edu

doi: https://doi.org/10.14740/jmc3447 seeking out extra-clinically prescribed pain medication. With that said, modern anesthetic developments, techniques and attitudes allow us to provide exceptional and safe anesthetic care while respecting the patient's autonomy and request for a narcotic-free anesthetic.

\section{Case Report}

We present a case of a 28-year-old G1P0 female at 39 weeks coming for a primary cesarean delivery due to breech presentation. The past medical history was significant for extensive substance use disorder. On initial pre-operative consultation, it was discovered that the patient had oscillated between extensive drug use and rehabilitation cycles for a dozen years, experimenting with multiple illicit substances, causing her family and husband a lot of anger and strife. She underwent extensive rehabilitation and behavioral therapy through the years and was able to stabilize on maintenance dose buprenorphone + naloxone combination lozenge (Suboxone ${ }^{\circledR}$ ) $8 \mathrm{mg} / 2$ $\mathrm{mg}$ daily. She had been on this stable dose for 2 years prior to discovering that she was pregnant and was seen in consultation 4 weeks prior to her scheduled cesarean delivery.

The patient's major concern was fear of receiving opioids and relapsing back into the addiction spiral. To appropriately plan, a large multidisciplinary team comprised of her addiction specialist, the in-house pain management physician, obstetrical anesthesiologist, the patient's obstetrician, and labor and delivery charge nurses was assembled. An exhaustive discussion was held that covered all narcotic, non-narcotic, regional and alternative options for the perioperative period. All options, possibilities and acceptable expectations were discussed at length. The patient's family was a major support structure and with her permission, they too were involved in the final planning. After two pre-operative visits, a plan was agreed upon and the cesarean delivery was scheduled.

The patient was the first case of the day and she took her daily Suboxone ${ }^{\circledR}$ maintenance dose. Her extended family and husband were present and provided immense support prior to her being transferred into the operating room (OR).

In the OR, standard American Society of Anesthesiologists (ASA) monitors were applied to the patient. She was properly positioned and an epidural was placed at the T8 level via loss of resistance to air technique. The catheter was threaded and a 
test dose was administered, which was negative for intravascular or intrathecal placement. This was followed by a spinal block with a 27-g pencil-point needle at the L4-L5 level. The $1.6 \mathrm{~mL}$ of $0.75 \%$ hyperbaric bupivacaine $(12 \mathrm{mg})$ with 200 $\mu \mathrm{g}$ epinephrine was administered. After confirming a bilateral T5 sensory spinal level, the cesarean section was uneventfully performed and a healthy baby was delivered.

After closure, the anesthesia team performed bilateral ultrasound-guided transverse abdominal plane blocks with a total of $15 \mathrm{~mL} 0.2 \%$ ropivacaine per side. The patient was then transferred to the post-anesthesia care unit and an infusion of $0.0625 \%$ of bupivacaine at $6 \mathrm{~mL} / \mathrm{h}$ was started in the epidural. She was also given ketorolac $15 \mathrm{mg}$ and acetaminophen 1,000 mg IV every $6 \mathrm{~h}$ for the next $24 \mathrm{~h}$.

The patient was seen the following morning for her postoperative check with her epidural having been running for 18 h. She had been out of bed to her chair, starting in the prior evening with minimal discomfort. Her IV ketorolac and IV acetaminophen were switched to oral ibuprofen $400 \mathrm{mg}$ and acetaminophen $650 \mathrm{mg}$ every $6 \mathrm{~h}$ in addition to her daily maintenance Suboxone ${ }^{\circledR}$. After expressing a desire to be free from the epidural, the infusion was paused, and then removed $4 \mathrm{~h}$ later, after experiencing minimal pain.

The remainder of the patient's stay was unremarkable. She went home on post-operative day 2 , having received no narcotics besides her maintenance Suboxone ${ }^{\circledR}$ dose throughout her entire perioperative stay. Her pain scores were minimal and she was very enthusiastic and satisfied with her care. She expressed immense gratitude and on serial follow-ups we were advised that the patient was doing great at home with no pain, not having taken any medication at all besides her daily maintenance Suboxone ${ }^{\circledR}$.

\section{Discussion}

With the ongoing epidemic of opioid abuse, the frequency with which anesthesiologists will encounter these patients in the perioperative setting will continue to increase. For women who are no longer using illicit substances, the fear of relapse is substantial and not to be taken lightly. This presents challenges for the anesthesiologist who must navigate patients through major painful surgeries while simultaneously respecting the patient's autonomy and wishes for a narcotic-free anesthetic.

Navigating a recovering polysubstance abuser through a narcotic-free anesthetic relies heavily on the use of regional anesthesia. Cesarean delivery is the most common surgical procedure performed in the United States accounting for roughly one-third of all deliveries. Post-cesarean pain management can be a considerable challenge, especially in those being treated for opioid dependence [3]. Adequate post-cesarean pain management couples regional or neuraxial blockade with a multimodal drug regimen. Scheduled administration of opioid-sparing adjuncts is recommended for all women post-cesarean delivery as they have an additive or synergistic benefit. Individually, nonsteroidal anti-inflammatory drugs (NSAIDs) have been shown to decrease opioid consumption by $30-50 \%$ and acetaminophen has been shown to decrease opioid consumption by $20 \%$ in healthy patients [4]. An observational study by Hoyt et al showed significant analgesia in patients who received an infusion of bupivacaine $0.1 \%$ with clonidine $1.2 \mu \mathrm{g} / \mathrm{mL}$ for $24-27 \mathrm{~h}$ postoperatively in opioid addicted parturients taking buprenorphine [5].

Other multimodal adjuncts have proven efficacious in abdominal surgery but have not been studied specifically in the obstetric population. Intraoperative glucocorticoids decrease narcotic requirement but may lead to hyperglycemia. Gabapentin has been used for multiple procedures but may have undesired maternal-fetal transfer. Ketamine has been used successfully for its narcotic-sparing property and aiding with withdrawal symptoms; however, a recent paper published by Williams suggests ketamine may activate the opioid system which may be less than ideal in populations seeking to avoid opioid medications [6].

Regional blocks beyond neuraxial techniques are likely to be the major source of analgesia. Transversus abdominis plane (TAP) block by itself or coupled with an ilioinguinal-iliohypogastric block has been used with success for postoperative analgesia [7]. More recently, the quadratus lumborum (QL) block has been utilized for abdominal procedures. A study by Mieszkowski showed a decrease in morphine consumption over $48 \mathrm{~h}$ coupled with lower intensity pain scores over the same amount of time for women given QL1 blocks after cesarean deliveries [8]. When comparing the TAP versus the QL block for cesarean section, Blanco et al found that the QL block significantly decreased morphine consumption over $48 \mathrm{~h}$ [9].

\section{Conclusion}

Above all, the decision and planning involves frank discussions between the patient and their anesthesiologist. Contemporary health care ethics is based on principles of autonomy, non-malfeasance, beneficence and justice. Patient autonomy is the hallmark of current medical decision-making, and it supplants the long-standing practice of paternalism epitomized by the Hippocratic Oath. Evolution of patient-centered decision making is credited to human rights principles espoused in the Nuremberg Code and the United Nation's Universal Declaration of Human Rights, among others [10]. In fact, American Medical Association's Code of Medical Ethics begins with this foundation in human rights: "A physician shall be dedicated to providing competent medical care with compassion and respect for human dignity and rights [11]". Patient autonomy respects patients' rights. Autonomy is fundamentally patientcentered asking "what does the patient want?" Therefore, the physician is a resource to assist the patient's informed, selfdetermined choice.

While these principles seem simple, clinical practice reveals more nuance. Patient autonomy is predicated on capacity, namely ability to comprehend the information and ability to evaluate consequences of a decision rationally [12]. Presuming capacity criteria are met, and the situation is free of coercion, then informed decision making further requires: 1) presentation of material information; 2) options and recommendation and 3) understanding by the patient [12]. Regarding these latter elements, innate bias of the physician can affect what is presented (or not) and how the options are framed regarding risks 
and benefits. In other words, clinical application of autonomy becomes more difficult because the physician might exclude some options or discount other options' valuation inappropriately relative to the patient's value system. Still, this bias must be overcome. One example, as discussed above, is when a patient requests a relatively narcotic-free anesthetic in situations where narcotic use is the perioperative norm.

\section{Acknowledgments}

None to declare.

\section{Financial Disclosure}

None to declare.

\section{Conflict of Interest}

None to declare.

\section{Informed Consent}

Obtained from patient by authors.

\section{Author Contributions}

David A. Gutman helped perform the described anesthetic, conduct background research and write up the case report. Jennifer R. Matos helped perform the literature search, write up the case report and perform numerous rounds of editing. Christopher A. Skorke helped perform the literature search, write up the case report and perform numerous rounds of editing.

\section{Data Availability}

The authors declare that data supporting the findings of this study are available within the article.

\section{References}

1. U.S. Department of Health and Human Services. The opioid epidemic numbers. https://www.hhs.gov/opioids/. May 102019.

2. Melemis SM. Relapse prevention and the five rules of recovery. Yale J Biol Med. 2015;88(3):325-332.

3. Hamilton BE, Martin JA, Osterman MJ, Curtin SC, Matthews TJ. Births: final data for 2014. Natl Vital Stat Rep. 2015;64(12):1-64.

4. Carvalho B, Butwick AJ. Postcesarean delivery analgesia. Best Pract Res Clin Anaesthesiol. 2017;31(1):69-79.

5. Hoyt MR, Shah U, Cooley J, Temple M. Use of epidural clonidine for the management of analgesia in the opioid addicted parturient on buprenorphine maintenance therapy: an observational study. Int J Obstet Anesth. 2018;34:67-72.

6. Williams NR, Heifets BD, Blasey C, Sudheimer K, Pannu J, Pankow H, Hawkins J, et al. Attenuation of antidepressant effects of ketamine by opioid receptor antagonism. Am J Psychiatry. 2018;175(12):1205-1215.

7. Young MJ, Gorlin AW, Modest VE, Quraishi SA. Clinical implications of the transversus abdominis plane block in adults. Anesthesiol Res Pract. 2012;2012:731645.

8. Mieszkowski MM, Mayzner-Zawadzka E, Tuyakov B, Mieszkowska M, Zukowski M, Wasniewski T, Onichimowski D. Evaluation of the effectiveness of the Quadratus Lumborum Block type I using ropivacaine in postoperative analgesia after a cesarean section - a controlled clinical study. Ginekol Pol. 2018;89(2):89-96.

9. Blanco R, Ansari T, Riad W, Shetty N. Quadratus lumborum block versus transversus abdominis plane block for postoperative pain after cesarean delivery: a randomized controlled trial. Regional Anesthesia Pain Medicine. 2016;41(6):7575-7762.

10. White SM, Baldwin TJ. The Human Rights Act 1998: Implications for anaesthesia and intensive care. Anaesthesia. 2002;57(9):882-888.

11. American Medical Association (AMA). AMA Code of Medical Ethics. https://www.ama-assn.org/sites/amaassn.org/files/corp/media-browser/principles-of-medicalethics.pdf. Revised: June 2001. Accessed: March 2020.

12. Waisel DB, Truog RD. An introduction to ethics. Anesthesiology. 1997;87(2):411-417. 